\title{
Letters
}

Website: bmj.com

Email: letters@bmj.com

\section{Medical errors and medical culture}

\section{There is no easy way around taking} responsibility for mistakes

EDITOR-The case commented on by Singer, Wu, Fazel, and McMillan is chilling in that the patient died in pain and suffering, and in the way it was handled by the senior attending physician-swept under the carpet, information falsified, and given a high minded sort of dismissal with "let this be a lesson." That is almost obscene.

The commentaries addressed most of the important points except discussing the fear of litigation and the fact that there are no easy answers when it comes to making mistakes. That needs to be said outright lest someone, especially someone in training who is less experienced, think that admitting a mistake stops at quality control or sharing responsibility, and that there is then some way around the difficult task of actually taking responsibility for the mistake.

Within the culture of medicine and even more broadly in modern society there seems to be a drive for finding the easy way out. In this case there is none, and it needs to be made very clear that this is a defining moment in the life of a physician with regard to integrity and professionalism. That must be included in the discussion of how a

\section{Advice to authors}

We prefer to receive all responses electronically, sent directly to our website. Processing your letter will be delayed unless it arrives in an electronic form.

We are now posting all direct submissions to our website within 24 hours of receipt and our intention is to post all other electronic submissions there as well. All responses will be eligible for publication in the paper journal.

Responses should be under 400 words and relate to articles published in the preceding month. They should include $\leqslant 5$ references, in the Vancouver style, including one to the BMJ article to which they relate. We welcome illustrations.

Please supply each author's current appointment and full address, and a phone or fax number or email address for the corresponding author. We ask authors to declare any competing interest. Please send a stamped addressed envelope if you would like to know whether your letter has been accepted or rejected.

Letters will be edited and may be shortened.

bmj.com

letters@bmj.com supervising physician deals with a trainee who has made a mistake, which was relayed with such insight and sensitivity by Wu.

Laurie Lyckholm assistant professor

Department of Internal Medicine, Virginia Commonwealth University, Box 980230,

Richmond, VA 23298-0230, USA

lyckholm@vcu.edu

1 Singer PA, Wu AW, Fazel S, McMillan J. Medical errors and medical culture. BMJ 2001;322:1236-40. (19 May.)

\section{Pain relief should have been provided} without hesitation

EDITOR-Large myocardial infarctions in elderly patients are often fatal, and whether or not earlier treatment would have resulted in the patient surviving will never be known. What can be said with certainty is that the patient mentioned in the education and debate section by Singer, Wu, Fazel, and McMillan suffered a terrible death, and with or without the results of the electrocardiogram the quality of the patient's life for the last five days could have been greatly improved. "On the next round the patient was still in severe pain ... We hesitated about whether to provide pain relief."

An uncommunicative patient is in severe pain, and the doctors hesitate to provide pain relief? Why, when the patient was so clearly, obviously, and distressingly in pain? Cautiously initiating treatment with opioids, and carefully adjusting the dose could have done much to have alleviated the patient's suffering. Such palliative treatment could also have done much to improve the patient's chance of survival.

As well as overlooking an errant electrocardiogram, an even more egregious error was the failure to observe a dictate that serves as the basis for compassionate care: To cure whenever possible. To comfort always.

Stephen Workman assistant professor

Dalhousie University, Department of Medicine,

Division of General Internal Medicine, Halifax

Nova Scotia, Canada B3H 2 Y9

sworkman@is.dal.ca

1 Singer PA, Wu AW, Fazel S, McMillan J. Medical errors and medical culture. BMJ 2001;322:1236-40. (19 May.)

\section{Move from"I" to "we" represents a} paradigm shift in responsibility

Editor-Singer, Wu, Fazel, and McMillan present a case of medical error and cover up with thoughtful commentary. ${ }^{1} \mathrm{Wu}$, however, inadvertently illustrates just how difficult to achieve the change in culture required to "learn to love mistakes" will be. He suggest how the doctor concerned should explain the mistake to the relatives of the woman who has died: "I regret to say that we made a mistake in your relative's care ... we missed signs of what was probably a heart attack ... I am devastated at being responsible for this, and can only tell you how sorry I am."

This statement illustrates both a desire to promote corporate responsibility for medical errors-we made a mistake-and a requirement that physicians take full personal responsibility for such-I am devastated at being responsible. I think that these two ways of seeing medical errors are incompatible; the move from "I" to "we" represents a paradigm change. The reconceptualisation of medical error as a corporate or "systems failure" necessitates a change in the way that physicians attribute guilt for error to themselves. In the case discussed the junior physician clearly believes responsibility for the error is his or hers.

This may well be a true belief. But it is not justified by the related facts of the case. A statement to relatives that "I am devastated at being responsible" would not be justified until the contribution of overwork, underfunding, and general NHS chaos had been investigated. If medical error is to be considered a failure of systems the justification for responsibility on the part of the physician could only arise as the result of investigation by the corporate entity involved. To encourage reporting of medical error the language of ethical commentary cannot drift between the "I" and the "we" paradigms. Ethicists must be consistent in their use of language in this debate. Corporate responsibility for medical error offers many advantages and is to be encouraged. It cannot operate unless physicians are encouraged to stop blaming themselves in a peremptory fashion for every calamity that befalls their organisation.

Wayne Lewis general practitioner Carreg Wen Surgery, Blaenavon, Gwent NP4 9AF wayne@drlewis.freeserve.co.uk

1 Singer PA, Wu AW, Fazel S, McMillan J. Medical errors and medical culture. BMJ 2001;322:1236-40. (19 May.)

\section{Changing the culture of blame requires a} revolution

EDITOR-I read with interest the series of articles on medical errors and medical culture by Singer, Wu, Fazel, and McMillan. ${ }^{1}$ I fear that without a revolution in the culture of blame within medicine things are unlikely to change. I believe the revolution we need is for individual doctors to be indemnified from personal blame after a medical mishap if the following conditions are adhered to. 
He or she, firstly, immediately communicates the mishap that has occurred to all necessary parties (including the patient); secondly, takes immediate necessary steps to neutralise or limit any harm; thirdly, then engages fully with critical analysis of the incident and the implementation of any remedial programme or training necessary.

The organisation for which that doctor works would remain accountable for the system under which the mistake occurred, leaving the patient with an appropriate avenue for financial redress, if justified. Should a pattern of multiple or recurring mistakes occur with a given doctor, this would still leave the employing organisation and any other interested party (General Medical Council, Commission for Health Improvement) with justification for action. This is not because of any individual error but because of a worrying pattern of apparent inability to learn from previous mistakes and benefit from remedial input.

Although I can offer this as an alternative vision, I am not clear how to achieve the seismic change in political, professional, and societal attitudes that would be necessary to move from our present position to the one I propose

Brendan Harrington consultant paediatrician Wrexham Maelor Hospital, Wrexham LL13 7TD

1 Singer PA, Wu AW, Fazel S, McMillan J. Medical errors and medical culture. BMJ 2001;322:1236-40. (19 May.)

\section{Global campaign to eradicate malaria}

\section{Meeting showed scope for scaling up campaign}

EDITOR-Yamey's editorial summarises some of the key issues raised at the fourth global partners' meeting for Roll Back Malaria. ${ }^{1}$ As he says, more and rapid action is desperately needed at country level. Three other messages, however, came across strongly at the meeting.

The first is that partners within countries are not waiting for the outside world to tell them how to start. Several countries have recognised the opportunities to make a real difference as part of Roll Back Malaria. For instance, in Uganda those responsible for malaria and for integrated management of childhood illness are working together to support the campaign's objectives instead of competing over territory.

After developing strategies to reduce poverty Uganda is already benefiting from increased malaria allocations through debt relief and other funds earmarked for poverty sensitive activities. Malawi is implementing a strategy for reducing the burden of malaria in pregnancy. Despite a relatively simple and cheap intervention known to be effective, few countries have gone this far. Tanzania has brought public and private partners together systematically to develop a national strategy for supplying insecticide treated nets and is achieving rapidly growing coverage rates.
These encouraging examples might well have happened without Roll Back Malaria, but they underscore the fact that great progress can be made in rolling back malaria. They also serve as a challenge to the campaign, showing that it has the opportunity to do much more than it has done to date.

The second message from the meeting was that many participants, including those from the world's poorest countries, were impatient at the slow pace of Roll Back Malaria. But countries have limited capacity to scale up quickly. Although more money is necessary to develop the needed capacity, and partners need to convert their pledges into resources, money is not the only essential ingredient. There also needs to be will to invest in people as the key to future success, by both governments and donors, and to create an environment where people can use their skills effectively.

The third important message from the meeting was that the power of communication can greatly increase the number and type of people willing and able to contribute. Strategies to optimise use of this resource will see Roll Back Malaria reaching those parts of the poorest countries that are always left till last.

Sylvia Meek director sylvia.meek@lshtm.ac.uk

Jane Edmondson human and institutional development coordinator

Malaria Consortium (London and Liverpool

School of Tropical Medicine), London School of Hygiene and Tropical Medicine, London WC1E 7HT

Dennis Carroll senior health adviser USAID Global Bureau, Office of Health and Nutrition, Washington, DC 20523, USA

1 Yamey G. Global campaign to eradicate malaria. $B M$ J 2001;322:1191-2. (19 May.)

\section{Malaria is paradigm of an emergent} disease

EDITOR-We believe that fundamental biological principles have been ignored in the debate over malaria and that eradication is not the objective of Roll Back Malaria, a campaign discussed in Yamey's editorial. We have posted a longer version of this letter as a rapid response (www.bmj.com/ cgi/eletters/322/7296/1191\#EL2). The Roll Back Malaria campaign seeks to halve child and maternal mortality due to malaria by 2010 . Any malaria control initiative mus learn lessons from the failed eradication programme of the 1950s and 1960s.

One of those lessons is that any chemotherapeutic, prophylactic, or insecticide based tool has a finite duration of efficacy: chloroquine and dicophane (DDT) rapidly induced resistance in Plasmodium spp and Anopheles spp respectively. These organisms reproduce rapidly and as vector/parasite systems have an unrivalled capacity to change, have coevolved an efficient hostparasite relationship, and are hugely diverse below the species level. Anopheles adapts rapidly to ecological, environmental, and climate change; such change is often local and operationally relevant to malaria con- trol. The development of drug and insecticide resistance and ecological and demographic change will outstrip the capacity of any health system to respond even if human resources were available to implement changes in policy on the basis of good evidence.

Resources available to public health services in sub-Saharan Africa have declined greatly over the past two decades; some countries are unstable or in active conflict. The poorest populations have limited access to health care; up to four fifths rely for the provision of malaria "control" on the uncontrolled informal sector. Health reform has ensured that no specific disease control budgets are available at district level, and HIV infection and tuberculosis have exacerbated the pressure on hospital services.

Impregnated bed nets are recognised to cut child mortality, but pyrethroid resistance is already present in west and southern Africa. No suitable alternatives are available, however, as multinational companies have concentrated on reducing pesticide use in agriculture by developing genetically modified monocultures and have reduced investment on the development of new pesticides for agriculture, which accounts for $90 \%$ of the market. Thus no investment will be made for new pesticides for public health alone.

Against this background-high biological diversity and adaptability in Anopheles spp and Plasmodium spp, limited human and financial resources at country level, ecological demographic and climate change, time limited efficacy of drugs and insecticidesthe likelihood that health systems change can outstrip the rate of biological change (a prerequisite for rolling back malaria) is not only optimistic but flawed. Malaria remains a paradigm of an emergent disease.

D H Molyneux professor, Lymphatic Filariasis

Support Centre

fahy@liv.ac.uk

G Barnish senior lecturer

Liverpool School of Tropical Medicine, Liverpool L3 5Q

S Looreesuwan professor

Mahidol University, Bangkok, Thailand

B Liese senior adviser

World Bank, Washington, DC 20433, USA

J Hemingway professor

Cardiff School of Biosciences, University of Cardiff, PO Box 915, Cardiff CF10 3TL

1 Yamey G. Global campaign to eradicate malaria. BMI 2001:322:1191-2. (19 May)

\section{Opioids in chronic non-malignant pain}

\section{Opioids can cause addiction even in patients with pain}

EDITOR-McQuay in his editorial says that we know that if the opioid sensitive pain later resolves treatment can be stopped without patients becoming addicts. ${ }^{1}$ Does he mean that there is little or no chance of addiction or that occasionally the patient will not become addicted? There is no reference given for this statement. 
As a medical student (long before evidence based medicine) I was led to believe that in this situation there was very little risk of addiction. But my faith in this comforting idea was shaken by my experience of being involved with the management of a mountaineer who had severe frostbite of the hands and feet in Nepal 40 years ago. When in hospital in Kathmandu the severe pain in his feet could only be controlled by opioids (pethidine). In discussions about the continued use of this drug I took a relaxed attitude because of the teaching I had received. The man later had to have both legs amputated below the knee. During this time he became thoroughly addicted to pethidine. The management of drug addition was less developed in those days and he decided to come off "cold turkey." His experience in achieving this is graphically described in his book, No Place for Man. ${ }^{2}$

From what we know of the effect of opioids in downregulating the opioid receptors it is hardly surprising that continued use of high doses of opioids even in opioid sensitive pain relief is likely to lead to addiction. The outcome, however, may well depend on the dose and route of administration. I agree with McQuay that we urgently need more hard data.

James S Milledge physician emeritus Northwick Park Hospital, Harrow HA1 3UJ

1 McQuay H. Opioids in chronic non-malignant pain. $B M$ 2001:322:1134-5. (12 May)

2 Mulgrew P. No place for man. London: Nicholas Vane, 1964.

\section{Chronic pain should not be undertreated}

EDITOR-I am a patients' advocate and literature researcher, not a physician. In internet community service work I have corresponded with hundreds of patients with chronic face pain. Many of these have diagnosed facial neuralgias or neuropathies. Many report that one or more doctors have refused to treat them with opioids, even on a trial basis. Some report having been accused of drug seeking behaviour simply for committing the offence of requesting treatment with drugs that they know from experience are effective for them. In the health insurance system in the United States the consequences of such a comment in a patient's medical record can be horrendous.

I recognise that treatment with opioids is generally less effective for the categories of pain that I see than for the general population. But from long exposure to online discussions between patients themselves, I know that some people do get relief from individual opioids or "cocktails" tailored by a pain specialist. I am forced by this experience to condemn outright the refusal of many medical professionals to even try such measures, in the absence of other effective medical or surgical remedies. I heartily endorse research to assess factors related to patients and efficacy of drugs, as suggested by McQuay. ${ }^{1}$

It is long past time to put to rest the myth that prescribed pain drugs create addiction problems on the street. This issue should be readily susceptible to simple retrospective studies. How many convicted drug offenders in the United States or United Kingdom have been prescribed opioids by a doctor? Surely these numbers are known or can readily be derived?

Drug offenders tend to come from population cohorts that are among the least served by medical caregivers. In the United States, the evidence is strong that medical practice for pain management is about to undergo a popular revolution. What a shame that the process had to be forced by patients' lawsuits, rather than proceeding from simple common sense and compassion on the part of professional caregivers. If you are one of those doctors who continue to withhold pain management measures from your patients, then I suggest that you need refresher training in current practice for pain management.

Richard A Lawhern network contact

Trigeminal Neuralgia Association (US), Sterling, VA 20165, USA

lawhern@erols.com 1 McQuay H. Opioids in chronic non-malignant pain. BMJ
2001;322:1134-5. (12 May.)

\section{Don't forget methadone for chronic pain}

EDITOR-McQuay in his editorial says that the use of opioids for chronic non-malignant pain can be messy, but this need not be so. The risks and benefits of opioids are well attested. The study of fentanyl patches versus long acting morphine is an imperfect comparison of one expensive opioid delivery system with another. ${ }^{2}$ McQuay chose manufacturers' recommendations over numerous clinical alternatives. Medical trials are often represented as a race with a clear winner. In this case, the winner happens to be the product of the company sponsoring the trial. McQuay's question on treating pain responsive to opioids presupposes that a patient has already tried opioids. We could instead ask whether doctors should deny opioids to a patient who seems to benefit from them? Withdrawing such drugs may be unwise or even unethical.

Differences between various opioids are to be expected because their effects are individual and doses never exactly comparable. Since this trial was not blind, the claim of modest advantages for fentanyl is not scientifically robust, as McQuay points out. Some reported improvements may also stem from the novelty factor, with a patch delivery system. Transdermal patches have certain benefits, but they also have problems. Dose adjustments are not easy, disposal can be hazardous, and adhesion can be a problem, especially in countries where people usually bathe daily. The choice of drug for chronic pain should not ignore the safety profiles of traditional opioids such as oral methadone, morphine, or codeine. From its use in addiction, methadone has exemplary long term safety data. It is also taken once daily. Although it is a cheap drug and perhaps of less interest to drug companies, methadone can be highly effective for chronic pain.

Clinicians should always consider the safest and most effective drug initially, moving to other options if problems arise. Cost is also a factor, especially in conditions requiring long term pharmacotherapy. Any stigma from methadone or morphine quickly vanishes when these drugs are used appropriately. Fentanyl patches should probably not be used as first line treatment. Likewise, long acting morphine, which is expensive and generally administered twice daily, should probably be second line treatment to methadone. If methadone is found to be unsatisfactory, buprenorphine, oxycodone, morphine (long or short acting), and fentanyl are all viable alternatives. Despite the best science, the use of such opioids is still often based on trial and error.

Andrew Byrne general practitioner

Drug and Alcohol, Redfern, New South Wales, 2016, Australia

$\mathrm{AB}$ makes a proportion of his income from treating addiction and pain management patients. No tobacco sponsorship. No cruel animal experiments performed in this practice.

1 McQuay H. Opioids in chronic non-malignant pain. BMJ 2001;322:1134-5. (12 May)

2 Allan L, Hays H, Jensen N-H, Le Polain de Waroux B, Bolt M, Donald R, et al. Randomised crossover trial of transdermal fentanyl and sustained release oral morphine for treating chronic non-cancer pain. BMJ 2001;322:1154-8.

\section{Early growth and coronary heart disease in later life}

\section{Analysis was flawed}

EDITOR-Eriksson et al concluded that in Finnish men born 60 years ago "low weight gain during infancy is associated with increased risk of coronary heart disease," yet they did not analyse infant weight gain. ${ }^{1}$ All their references to infant growth relate to size at 1 year (table 3 ). Had they applied the key regression models that we have described $^{2}$ to separate the effects of weight at different ages on later outcome, they would have found that infant weight gain was unrelated to risk of coronary heart disease.

In their simultaneous analysis the hazard ratios for birth weight and weight at 1 year were similar and less than 1, showing that greater weight during infancy is protective. Weight gain is weight at 1 year less weight at birth, so if weight gain were protective it would appear as a protective effect of weight at 1 year and a relatively deleterious effect of weight at birth. ${ }^{2}$ But the two effects were equally protective, so weight gain in infancy (strictly, upwards centile crossing) is unrelated to later coronary heart disease.

The hazard ratios for weight at birth and at 1 year can be rearranged as hazard ratios for mean weight and weight gain. The hazard ratio for weight gain is equal approximately to the square root of the ratio of the hazard ratios at 1 year and at birth-that is, $\sqrt{0.84 / 0.94}=0.95$. This is similar to the birthweight hazard ratio, which was not significant (95\% confidence interval 0.83 to 1.06).

During childhood, increasing fatness was related to increased risk of coronary heart disease, particularly in those who were initially thin. ${ }^{1}$ This corresponds to our interaction model. ${ }^{2}$ The hazard ratio for the change in body mass index from age 1 to age 12 is obtainable from our combined 
model. The hazard ratios for body mass index at ages 1 and 12 are 0.83 and 1.03 (table 4). On the assumption that they would be similar if fitted simultaneously, the square root of the ratio of hazard ratios gives the approximate hazard ratio for the change in body mass index, $\sqrt{1.03 / 0.83}=1.11-$ not that different from 1.20.

So we agree that infant thinness and subsequent increasing fatness are synergistic risk factors for coronary heart disease, as others have shown. ${ }^{3}$ But for centile crossing to relate to coronary heart disease the hazard ratios for body size at the start and end of the period should differ significantly, and this is not the case in infancy. Routine use of our approach ${ }^{2}$ would have avoided this confusion.

T J Cole professor of medical statistics Centre for Paediatric Epidemiology and Biostatistics, Institute of Child Health, London WC1N 1EH

tim.cole@ich.ucl.ac.uk

M Fewtrell MRC senior clinical scientist

A Lucas MRC clinical research professor

MRC Childhood Nutrition Research Centre,

Institute of Child Health

1 Eriksson JG, Forsén T, Tuomilehto J, Osmond C, Barker DJP. Early growth and coronary heart disease in later life: DJP. Early growth and coronary heart disease in later

2 Lucas A, Fewtrell M, Cole TJ. Fetal origins of adult Lucas A, Fewtrell M, Cole TJ. Fetal origins of ad
disease-the hypothesis revisited. BMJ 1999;319:245-9.

3 Frankel S, Elwood P, Sweetnam P, Yarnell J, Smith GD.

Birthweight, body mass index in middle age and incident Birthweight, body mass index in middle age and
coronary heart disease. Lancet 1996;348:1478-80.

\section{Authors' reply}

EDITOR-Cole et al are wrong. Coronary heart disease is clearly related to low weight gain during infancy in addition to low birth weight. Conditional on birth weight, the additional predictive power of infant weight gain is expressed by a $\chi^{2}$ statistic of 9.26 $(\mathrm{P}=0.002)$. In a simultaneous analysis the hazard ratio for a one standard deviation decrease in birth weight is $1.29 \quad(95 \%$ confidence interval 1.14 to $1.45, \mathrm{P}<0.001$ ) and for a one unit decrease in standard deviation scores for weight between birth and age 1 it is 1.21 ( 1.08 to $1.36, \mathrm{P}=0.001$ ). The mistake that Cole et al make is in parameterising the model so that part of the effect of infant weight gain is lost in an average weight term.

It is not adequate to analyse data on birth weight and weight at age 1 using what they describe as key regression models. These are dependent on assumptions of linearity. In the analyses of data from Hertfordshire, which first established the link between coronary heart disease and low weight gain in infancy, it was necessary to develop a more complicated model and express the results by using contours of disease risk. ${ }^{1}$ The Helsinki study provides a striking replication of these results and also allows us to examine the effects of growth through childhood. In our paper we focused on the finding that the effects of childhood weight gain on later coronary heart disease are conditioned by ponderal index at birth (birth weight/length ${ }^{3}$ ).

Because the Helsinki dataset includes an average of nine measurements of height and weight during infancy for 8760 men and women we can now pinpoint the time in infancy when growth faltering begins and relate this to infant feeding, housing conditions, family size, and other variables. The study allows, for the first time, detailed description of the paths of fetal, infant, and childhood growth that precede the development of chronic diseases in later life. When these descriptions are published, would-be commentators on our analyses will be welcome to have any additional data needed for clarification. This will avoid the kind of erroneous conclusions that have been drawn by Cole et al.

C. Osmond medical statistician

D J P Barker professor of clinical epidemiology

MRC Environmental Epidemiology Unit

(University of Southampton), Southampton

General Hospital, Southampton SO16 6YD

co@mrc.soton.ac.uk

J G Eriksson senior researcher

T Forsén research fellow

National Public Health Institute, Department of

Epidemiology and Health Promotion, Diabetes and

Genetic Epidemiology Unit, FIN-00300 Helsinki,

Finland

1 Barker DJP, Osmond C, Winter PD, Margetts B, Simmond SJ. Weight in infancy and death from ischaemic heart disease. Lancet 1989;ii:577-80.

\section{Riluzole for motor neurone disease}

\section{Reply from chairman of appraisal committee at NICE}

EDITOR-In commenting on the National Institute for Clinical Excellence's guidance on riluzole Sandercock et al show the difference between assessment and appraisal of evidence. ${ }^{1}$ One form of evidence used in an appraisal is a formal systematic review. The assessment report does not make recommendations on how the technology should be used in the NHS; that is the job of the institute's appraisal committee. The committee also receives submissions from patient and professional organisations, which provide perspectives not captured by a formal review of published evidence.

The committee takes account of the clinical need of patients and the broad balance of benefits and costs of the technology. Its conclusions are subject to consultation and can be appealed against. This is all some distance on from the original assessment of the evidence.

Sandercock et al suggest that a superficial reading of the guidance may not give an adequate understanding of the evidence base for riluzole. The institute's guidance identifies what the committee considered to be important elements of the evidence. The full assessment report is available on the institute's website (www.nice.org.uk). Neither document, however, can fully convey the depth of the committee's consideration of the evidence.

Sandercock et al advise clinicians to prescribe in accordance with riluzole's licence, say that patients offered the drug should be fully informed, and suggest further research.
This is fully in accordance with the institute's guidance.

In the same cluster of letters Wheatley and Gray accuse the institute of recommending a treatment "when there is no significant evidence of benefit." Although the statistical measures of benefit may not be great (we acknowledge that the relative hazard reduction for tracheostomy free survival is $12 \%$ (that is, $1.00-0.88$ ), not $17 \%$ as quoted in the guidance), the committee is required to consider what the reported measures of clinical effectiveness of the technology actually mean to people with the disease.

Wheatley and Gray say that the guidance is contrary to the conclusions of the Sandercock report. We do not look to the authors of assessment reports for conclusions as, unlike the appraisal committee, they have neither access to the full evidence base nor the range of skills necessary to undertake an appraisal.

The institute's guidance need not deter further research into this disease or its treatment. The guidance sets out a clear research agenda, which we would encourage the manufacturer and clinicians to pursue.

David Barnett chairman of appraisal committee National Institute for Clinical Excellence, London WC2N 5HR

nice@nice.nhs.uk

1 Correspondence. Riluzole for motor neurone disease. BMJ 2001;322:1305-6. (26 May.)

\section{Any placebo controlled trial of riluzole would surely be unethical now}

EDITOR-We are concerned about the opinions expressed by Sandercock et al regarding the clinical efficacy of riluzole for the amyotrophic lateral sclerosis form of motor neurone disease and the appropriateness of the guidance issued by the National Institute for Clinical Excellence (NICE).

Riluzole has been subject to regulatory scrutiny by the European Agency for the Evaluation of Medicinal Products and the Food and Drug Administration; an independent review by the Cochrane Collaboration$^{2}$; and the review by the National Institute for Clinical Excellence. The health technology assessment report by Sandercock et al formed only part of the evidence based assessment by the institute; evidence from a wide range of expert clinical, research, and patient based sources was also made available.

Of particular importance are the results of two large prospective, randomised, double blind placebo controlled trials of riluzole in amyotrophic lateral sclerosis. ${ }^{3}{ }^{4}$ The study by Bensimon et al $(\mathrm{n}=155)$ was stopped at 18 months because of a clear difference in favour of the active treatment arm. ${ }^{3}$ As a result, patients taking placebo were offered active treatment, which meant that the authors were unable to determine overall survival in comparison with survival with placebo. However, analysis of the study to 18 months showed that patients taking riluzole had a $28 \%$ better survival rate than those taking placebo $(\mathrm{P}=0.014)$. 
The second study, by Lacomblez et al $(n=959)$, reported that after adjustment for prognostic factors the risk of death or tracheostomy at 18 months was $35 \%$ lower with riluzole $100 \mathrm{mg} /$ day than with placebo $(\mathrm{P}=0.002){ }^{4}$ This study also assessed the clinical efficacy and safety of different doses of riluzole and concluded that $100 \mathrm{mg}$ was the optimal daily dose $(\mathrm{P}=0.003)$. A closer investigation of the data showed that the significant effect of riluzole was observed in earlier disease stages. ${ }^{5}$

These data provide the evidence that riluzole is clinically proved to extend survival in amyotrophic lateral sclerosis. In a disease with a prevalence of 4/100 000 and in which average life expectancy is 36 months, clinical trials looking at over 1000 patients $^{34}$ over 18 months are a reliable basis on which to establish clinical effect. Sandercock et al suggest that future clinical trials should include a placebo, but we take the view that any placebo controlled trial is unethical once clinical efficacy has been clearly shown.

The important information that Sandercock et al fail to report is that riluzole is the only clinically proved treatment available to patients with amyotrophic lateral sclerosis and might extend survival.

Martin Ford United Kingdom medical director Cyrille Leperlier international medical director Aventis Pharma, Aventis House, West Malling ME19 4AH

1 Sandercock J, Burls A, Hyde C, Fry-Smith A, Barton P, Bryan S, et al. Riluzole for motor neurone disease. $B M J$ 2001;322:1305. (26 Mav.)

2 Miller RG, Mitchell JD, Moore DH. Riluzole for amyotrophic lateral sclerosis (ALS)/motor neuron disease (MND). In: Cochrane database of systematic revieus Isseas 3 Oxford: Oxford Software, 2000. (www.nihs.go.jp/acc) Oxford: Oxford Software, 2000.

cochrane/revabstr/ab001447.ht.) , in 1994:330:585-91.

4 Lacomblez L, Bensimon G, Leigh PN, Guillet P, Meininger V. Dose-ranging study of riluzole in amyotrophic latera sclerosis. Lancet 1996;347:1425-31.

5 Rivière M, Meininger V, Zeisser P, Munsat T. An analysis of extended survival in patients with amyotrophic lateral sclerosis treated with riluzole. Arch Neurol 1998;55:526-8.

\section{Antidepressant trials generally have methodological defects}

EDITOR-The study reported by Woelk tried to show the antidepressant properties of St John's wort, ${ }^{1}$ but its methodology was subsequently much criticised. ${ }^{2}$ We put the four major criticisms together to form a short "methodological quality" checklist and added one item regarding the integrity of blindness: the checklist assessed use of remission criteria; use of a three arm design; use of an active placebo; individual determination of the dose of the reference compound; and evaluation of the integrity of blindness.

We have stated that blindness is a major defect in antidepressant trials and that it has often jeopardised their validity. ${ }^{3}$ In the study reported by Woelk, because of the tolerance profile of imipramine it was probably easy for the patients (and evaluators) to guess whether they were receiving St John's wort or imipramine. The expectations of both patients and evaluators may thus have biased the results.

To check whether the criticisms about the study reported by Woelk were addressed in "regular" antidepressant trials we performed a Medline search for the year 2000. We identified 19 randomised controlled trials that evaluated the short term efficacy of regular antidepressants in major depression and reviewed them and rated them according to the above checklist. Two of the studies addressed three requirements in the checklist, five addressed two, and 12 addressed one or none. Interestingly, these 12 studies did not elicit any critical correspondence in the three issues of the journals that followed publication. In conclusion, many trials that evaluated the efficacy of regular antidepressants bore the limitations observed in the study reported by Woelk.

If the criticisms against the study reported by Woelk are correct then St John's wort still needs to prove itself. But this may also hold for some regular antidepressants. The general methodological defects of antidepressant trials smooth out the differences in the efficacy of antidepressants and lead to all antidepressants being lumped into a single category regardless of their efficacy. But the compounds that have shown their efficacy in high quality trials (for example, those with a three arm design) should not be lumped with those that have not.

Only better designs and methods could enable discrimination between compounds that were and were not efficacious; this holds for new and alternative compounds as well as for classic ones.

Christian Even praticien hospitalier evenlafitte@compuserve.com

Serge Friedman attaché de consultation Roland Dardennes praticien hospitalier Hôpital Sainte-Anne, Clinique des Maladies Mentales et de l'Encéphale (Service du Pr Guelfi) Université Paris V, 75674 Paris Cedex 14, France

1 Woelk $\mathrm{H}$ for the Remotiv/Imipramine Study Group. Comparison of St John's wort and imipramine for treating parison of St John's wort and imipramine for treating
depression: randomised controlled trial. $B M J$ depression: rand

Correspondence. Comparison of St John's wort and imipramine. BMJ 2001;322:493-4. (24 February)

3 Even C, Siobud-Dorocant E, Dardennes RM. Critical approach to antidepressant trials. Blindness protection i necessary, feasible and measurable. $B r J$ Psychiatry 2000;177:47-51.

\section{Doctors should tell patients truth about their waiting lists}

EDITOR-Hayward advocates getting angry on behalf of our patients and complaining openly about the inadequacies of the services we offer. ${ }^{1}$ As the oncologist who went public on the effects of excessive waiting lists for radiotherapy, ${ }^{2}$ I would like to defend myself against his criticism that it took me so long.

It is obvious that untreated cancers will grow, and waiting lists for radiotherapy have been a problem for some time throughout the United Kingdom. It was only when Edwards and I completed our audit, however, that the full effects became clear. My only hesitation in publishing was in con- tacting my defence union and the Royal College of Radiologists, without whose support I would have been nervous of the management's response.

Unfortunately, although the paper caused a brief national stir with cries for more resources, a year later the waiting list for radiotherapy in Glasgow is no shorter. The difference is that I now tell patients there is a risk that their potentially curable lung cancer may progress and become incurable while they are on our waiting list. The patients know that I am angry on their behalf, but that is little consolation to either them or me when I have added to their already considerable distress and anxiety by telling them the truth about the waiting list.

I agree with Hayward that doctors should complain when the service fails patients-but not to improve our status, rather to enlist the patients' support so that together we have more chance of effecting change. Meanwhile, the truth is more painful for both patients and doctors to face than for the managers and politicians, who ought to be accounting for the inadequacies of the NHS.

Noelle O'Rourke consultant in clinical oncology Beatson Oncology Centre, Western Infirmary, Glasgow G11 6NT norourke@tinyworld.co.uk

1 Hayward R. Doctors on the ropes. BMJ 2001;322:805. (31 March.)

2 O'Rourke N, Edwards R. Lung cancer treatment waiting times and tumour growth. Clin Oncol 2000;12:141-4.

\section{Equipoise is not synonymous with uncertainty}

EDITOR-I was surprised by Sackett's contribution in the cluster of letters about clinical equipoise. ${ }^{1} \mathrm{He}$ attacks the word equipoise, on the ground that it is not used as commonly as the alternative word, uncertainty. Uncertainty, unlike equipoise, covers a range of situations, not just clinical trials. It is the meaning behind the word that is important.

Equipoise has been clearly defined within the paradigm of expected utility theory. "Patient equipoise" applies when the expected utilities of comparator treatments are equivalent. This provides a clear and precise meaning to the word equipoise, but if someone does not like the word then he or she should substitute another word with the same meaning. Uncertainty cannot be that word. Uncertainty is the opposite of certainty and therefore covers a huge range of possibilities, from equipoise all the way to certainty.

Gifford makes a relevant point in the same cluster of letters, highlighting the difference between the conditions under which a trial may be conducted and those that are necessary for participation of individual patients. ${ }^{1}$ So Sackett is right when he says that uncertainty is a perfectly appropriate criterion on which to mount a trial. However, Gifford is right in saying that the amount of evidence required for a policy decision (for example, to approve a new treatment) is much greater than that 
required for individual patients to choose their treatment (or for the doctor to do so if the patient cannot take part in the decision). Sackett is right when he says that uncertainty is the most widely used term, and it can describe the conditions under which a trial can ethically proceed. However, patient equipoise or some similar term is needed to describe the conditions under which patients should rationally accept randomisation.

When I counselled patients about amniocentesis and chorionic villus sampling I did not just say that I was uncertain about their effects but gave a range of probabilities within which I believed the true effect would lie, and I disclosed my best prior estimate within this range. I said that amniocentesis would cause miscarriage in 1 case in 200 while chorionic villus sampling had between 1.5 and 4 times this risk and that my best prior guess was that it would be twice as risky. As a result, some patients chose amniocentesis, some (mostly people at high genetic risk) chose chorionic villus sampling, and a few were unable to decide-they were in personal equipoise and went into the Medical Research Council's trial comparing the two techniques.

R J Lilford professor of clinical epidemiology University of Birmingham, Birmingham B15 2TT R.J.LILFORD@bham.ac.uk

Correspondence. Uncertainty about clinical equipoise BMJ 2001:322:795-6. (31 March.)

2 Edwards SJL, Lilford RJ, Braunholtz DA, Jackson JC, HewiEdwards SJL, Lilford RJ, Braunholtz DA, Jackson JC, Hewi-
son J, Thornton J. Ethical issues in the design and conduc of randomised controlled trials. Health Technol Assessment 1998;2(15)

\section{Medical litigation}

\section{Legal aid is still needed}

EDITOR-Barton is right to draw atttention to the inappropriate use of legal aid, for bad cases with poor lawyers. ${ }^{1}$ However, although it is true that after the event insurance can deliver a service more likely to take up only the stronger cases, there will still be a need for careful appropriate legal aid.

I work as an expert witness for both defence instructed cases and claimant cases. I have no axe to grind and no real interest in who is footing the bill. What I do know is that carefully prepared cases can succeed and that this takes enormous time and effort on the part of the solicitors involved. Claimants start at a huge disadvantage, with no records, poor medical knowledge, and no legal knowledge. If state funding is to be used to secure justice one has to accept the need for the disadvantaged to receive speedy, good, and appropriate advice.

Both privately funded insurance cases and well screened publicly funded cases can and do coexist-ideally those who can afford insurance to cover their legal fees should use them and those who can't should remain able to use legal aid.

Adrian Rogers private principal and NHS non principal

Exeter EX2 4EG

A@DrAdrianRogers.co.uk

1 Barton A. Medical litigation: Who benefits? $B M$ J 2001;322:1189. (12 May.)

\section{The ex-barrister's tale}

EDITOR-I agree with Barton's personal view on medical litigation, ${ }^{1}$ and I comment on the basis of my experiences as a barrister in independent practice before I became a principal in general practice. The conflict of interest pointed out by Barton is correct and obvious to outsiders. But lawyers often use denial and rationalisation to justify their practices, perhaps without even realising that they are doing so.

As a barrister, when I was advising on the merits of cases, I tried to give an hones and realistic opinion about their prospects of success. This, however, did not go down well with my legal colleagues. The comments I received included, "the solicitor isn't going to like this," "you are too judgmental," "you need to give a good estimate of the prospects of success or the client won't get legal aid," and "it's impossible to quantify the prospects of success, as any idiot knows." I did not find my own legal practice congenial or profitable (perhaps because I was an idiot) so I gave it up and returned to medicine. A case of survival of the unfittest? Peter Gray general practice principal Sittingbourne, Kent ME10 1ND pgray@gn.apc.org

1 Barton A. Medical litigation: Who benefits? BMJ 2001;322:1189. (12 May.)

\section{Career advice for doctors with a chronic illness}

\section{Occupational health service can provide help}

EDITOR-MacDonald raises numerous important issues in her editorial about doctors with chronic illness. ${ }^{1}$ I wish to make several comments about her article and clarify the role of occupational health in helping doctors with chronic illness.

The role of occupational health services is varied; but the advice provided is impartial and given in the health interests of the person affected. A key task is to advise on rehabilitation and placement in suitable work of those temporarily or permanently disabled by sickness or injury. ${ }^{2}$ Most occupational health professionals strive to help people remain in useful work by advising on suitable modifications to their duties. Sometimes the medical condition impairs a person's function to an extent that job modifications are impractical, and in these circumstances retirement because of ill health may be advisable.

MacDonald also mentions several difficulties that doctors with a chronic illness may face in their careers; such as inflexible working patterns, poor contingency cover, and colleagues who are sympathetic until it affects them. These difficulties can be managed, and solutions can be implemented when the problems are addressed properly by a professional management structure. I have found medical directors of doctors extremely understanding and sym- pathetic when they receive professional advice on a doctor with chronic illness from an occupational health service. Good rehabilitation should be directed at maximising a person's contribution at work.

The Disability Discrimination Act 1995 defines the legal meaning of disability, but occupational physicians should not agonise over whether a person is disabled under the act or not. This decision is one made by the members of an employment tribunal. ${ }^{3}$ An occupational physician, when dealing with a doctor with long term illness, will assess the person clinically and then understand the activities of the job. After this the occupational physician will concentrate on advising on suitable adjustments to the work of the doctor to help him or her undertake the work.

Doctors with chronic illness do face some job difficulties; the best way to manage the situation is for people in a management position to address it in a professional fashion. The managers are likely to require specific advice on the individual doctor from an occupational physician to manage the situation in the best way. This process is likely to achieve best outcome for the individual doctor and also for the employing organisation.

Ian Aston consultant occupational physician Queen's Medical Centre, Nottingham NG7 2UH

1 MacDonald, R. Career advice for doctors with a chronic illness. BMJ 2001;322:1136-7. (12 May.)

2 BMA Occupational Health Committee. The occupational physician. 4th ed. London: BMA Publications, 1994:5.

3 Vicary $v$ British Telecommunications plc [1999] IRLR 680.

\section{You have to do whatever it takes to achieve your goal in medicine}

EDITOR-I thank MacDonald for raising the issue of chronic illness in doctors. ${ }^{1}$ I note, however, that the four case studies provided were all new presentations of illness, and no views were offered from those doctors having undertaken their training and career with an existing chronic illness.

I am a doctor who has had chronic renal failure from birth (congenital urethral valves leading to reflux nephropathy). As a child, having spent many months in hospital, I entered school aiming to be a doctor. Aged 18 , I achieved my entrance grades for medical school as the last of my native kidney function petered out. I simultaneously started haemodialysis and my first year at university.

After 18 months I received my first renal transplant. Although thereafter I was biochemically well, owing to the side effects of immunosuppressive drug treatment (principally from corticosteroids for Cushing's syndrome), it was one of the unhappiest times of my life. Successful treatment of patients does not always parallel quality of life. After three years the graft failed, and I entered my final year having continuous ambulatory peritoneal dialysis. I continued my regimen of 30 minute dialysis exchanges, four times a day, every day through my final Master of Biology exams, junior and senior house officer posts (in casualty and orthopaedics), and freely went skiing, snowboarding, and sailing more than ever. 
After four years of continuous ambulatory peritoneal dialysis I received my second renal transplant, and in the four years since then I have been in anaesthesia training in the United Kingdom and Australia. My ultimate achievement to date was to be the anaesthetist for a consultant paediatric urologist, who, 29 years earlier, had been the urology registrar assisting during my own nephrectomy when I was 1 year old (he did not recognise me).

I have had serious medical problems and complications along the way, and in time this graft will fail and I will continue on my cycle of dialysis and transplantation. But as I have had a chronic illness since birth it has not been enough for me to just cope, take six months to sort myself out, or find a niche for myself which matched my health needs and allowed me to carry on working. Experience with a physical disadvantage has taught me that you have to do whatever it takes to achieve your goal in medicine.

Julian Berry specialist registrar anaesthetics and intensive care, South-West School of Anaesthesia Department of Anaesthetics and Intensive Care, Treliske Hospital, Truro, Cornwall TR1 3LJ jmcberry@hotmail.com

1 MacDonald, R. Career advice for doctors with a chronic illness. BMJ 2001;322:1136-7. (12 May.)

\section{Drugs are not best for obesity}

EDITOR-Després in his editorial recommends more studies that test drug treatment for obesity. ${ }^{1}$ Most doctors and patients would agree that the medical management of obesity is a failure. The modern epidemic of obesity implies that there is an environmental rather than a metabolic cause promoting prolonged positive energy balance. Treatment should therefore move more into the field of behavioural psychology, ${ }^{2}$ using paradigms taken from treatment of addictive behaviours to promote a healthier lifestyle. ${ }^{2}$ Doctors still have an important role in encouraging exercise and eating habits, rather than prescribing potentially dangerous "magic bullets."

In this light, the editorial by Després, in which he advocates long term drug treatment for obesity (and when should you stop?), makes poor sense both medically and economically. It is counterproductive and uneducational, deceiving patients into thinking that they have a disease that can be treated with a drug so that they need not make any effort to change the poor lifestyle that is at the root of obesity. The behavioural approach to obesity is as successful as that of drugs $^{2}$ and has minimal side effects, but it is obviously less attractive for research support from industry. It is, however, surely the direction in which professionals must go to try to lessen the tremendous health burden of obesity. $^{5}$

Elliot M Berry senior physician

Department of Human Nutrition and Metabolism, and Internal Medicine, Hebrew

University-Hadassah Medical School, Jerusalem,

Israel, 91120

Berry@md.huji.ac.il
1 Després J-P. Drug treatment for obesity. BMJ 2001;32:1379-80. (9 June.)

2 Wing RR, Goldstein MG, Acton KJ, Birch LL, Jakicic JM, Sallis Jr JF, et al. Behavioral science research in diabetes: lifestyle changes related to obesity, eating behavior, and physical activity. Diabetes Care 2001;24:117-23

3 Prochaska JO, DiClemente CC, Norcross GC. In search of how people change. Am Psychol 1992;47:1102-14.

4 Rollnick S, Heather N, Bell A Negotiating behavio

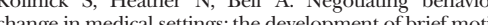
change in medical settings: the development of brief mot-

5 Thompson D, Edelsberg J, Colditz GA, Bird AP, Oster G. Lifetime health and economic consequences of obesity. Arch Intern Med 1999;159:2177-83.

\section{I was obviously fortunate when my salivary gland was removed}

EDITOR-Prior reports that she saw seven surgeons in all when she had a salivary gland removed. ${ }^{1}$ When I had one removed I was lucky. Firstly, my dentist sent me to St George's Hospital in London, where I saw a helpful registrar (surgeon 1). Secondlyafter various proddings, computed tomography, and a surreal $x$ ray examination, when I was the only patient not handcuffed to a prison warder-an operation was decided on quickly.

The operation was at 8 am on a Monday morning, and I was admitted to hospital at 7 am, which meant I slept in my own bed and saved the NHS the cost of a bed for a night. My surgeon was the epitome of courtesy and helpfulness. Why not name names? He was Professor John Langdon.

I was warned to expect at best temporary facial paralysis and at worst permanent paralysis (which they would try to repair). By then I knew I had a tumour, possibly lymphoma, that embraced my facial nerve. I asked that, if the nerve needed severing, this would not be intentionally done on this occasion. Professor Langdon's expression told me that the idea was unthinkable.

Surgeon 3 took the ward round on the morning after the operation and, needing to know how well I could grimace, asked me to show him my teeth. I reached towards a plastic dish, but he didn't get the joke. Professor Langdon didn't work at St George's on Tuesdays but nevertheless drove across London to see me. He didn't have to, and it was very good of him.

Surgeon 4, a house doctor, took out 40 or so careful stitches a week later-on Boxing Day. He wouldn't tell me the pathology results as he wasn't allowed to; no one senior enough to tell me was available. He refused my suggestion that he go out of the room, leaving my notes open at the pathology report. I thought this was a trifle pedantic of him, but then pedantry is often the preserve of the young.

I had good, courteous, and informative treatment; the atmosphere in the department reflected the high standards set by its senior members.

Caroline Richmond freelance journalist

11 Kennet Close, London SW11 2DG

c@roline.demon.co.uk

1 Prior P. A tale of seven surgeons. BMJ 2001;322:1433. (9 June.)

\section{A poem to benefit health}

EDITOR-Smith asks why doctors are so unhappy. ${ }^{1}$ Altogether 17 letters were printed in the $B M J$ after his editorial. ${ }^{2}$ They included one from Laurence saying that doctors need to be adaptable to change and one from Jakeman saying that doctors should concentrate on positive aspects of their work. ${ }^{2}$ Davies thought that we could be victims of our misperception of the world and need to have the courage to rediscover our own happiness.

These problems and the suggestions are not new. As Epictetus noted in the 4th century BC, "men are disturbed not by things but by the views they take of them." Centuries later John Keats asked, "Do we retreat from the reality of the outer world into ourselves at times, or do we retreat from the pressures of the outside world into the reality of our inner selves?" In 1993 the doctor-poet Dannie Abse, musing on this, commented that "imaginative daydreaming is an escape from the precipitous pessimism of living or dealing with problems and the sphere of sorrow, and it is used to restore balance."

Colleagues and I have asked the question, "Could or does reading or writing poetry benefit health?" Given the affirmative response, ${ }^{5}$ the following poem may help unhappy doctors.

\section{The tides of change}

In tides of change

Seas turn sands shift

Winds blow storms brew

People posture and position

Pose and preen with a moving scene;

Then when it seems that waters rage

And dirt and mud are outwards flung

Change tack to ward attack

Pause to plan and think it through

Duck dive parry strive to survive;

Take a chance laugh and start to branch

Learn to flex flow

Cut thrust bob bounce

To stay afloat to beat and dam

The tides of change.

Robin Philipp consultant occupational physician Department of Occupational Medicine, Bristol Royal Infirmary, Bristol BS2 8HW Robin.Philipp@ubht.swest.nhs.uk

1 Smith R. Why are doctors so unhappy? BMJ 2001;322:1073-4. (5 May.)

2 Correspondence. Why are doctors so unhappy? BMJ 2001;322:1361-5. (2 June.)

3 Philipp R, Baum M, Mawson A, Calman K. Humanities in medicine beyond the millennium. London: Nuffield Trust, 1999. (Nuffield Trust series No 10.)

4 Philipp R, Coppell C, Freeman H. Poetry and the art of medicine. BMJ 1994;308:63.

5 Philipp R, Robertson I. Poetry helps healing. Lancet 1996;347:332-3.

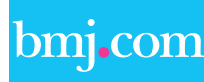

Rapid responses

Correspondence submitted electronically

is available on our website 